\title{
ORT.02 - Hepatitis E virus prevalence among chronic kdney disease hemodialysis-dependent patients
}

Andreza Salvio Lemos ${ }^{1 *}$; Camilla Rodrigues de Almeida Ribeiro ${ }^{1}$; Jakeline Ribeiro Barbosa ${ }^{1}$; Danielle Malta Lima ${ }^{2}$; Jeová Kenny Baima Colares ${ }^{2}$; Livia Melo Villar ${ }^{1}$; Vanessa Salete de Paula ${ }^{1}$.

1 Fiocruz - Fundação Oswaldo Cruz;

2UniFor - Universidade de Fortaleza;

Introduction: Hepatitis E virus (HEV) is responsible for acute self-limiting disease worldwide, mostly among immunocompetent person. However it can be manifested in different forms, according to the immune state of the patient. A relevant group for studies of HEV infection is the chronic kidney disease hemodialysis-dependent (HD) population, which may be lead to a transplant and immunosuppressive treatment, mainly with tacrolimus and its analogues, which have been described as related to chronic hepatitis E. Also this special group has been reported to present higher prevalence in comparison to immunocompetent populations in HEV-GT3 (HEV -Genotype 3) circulating countries. In Brazil, the prevalence of HEV is still underestimated among $\mathrm{HD}$ patients.

Objective: Elucidate the HEV prevalence and circulation among HD patients in Brazil.

Methodology: To elucidate the prevalence, 286 plasma samples were collected between 2013 and 2015 from three private kidney treatment centers: 2 located in the Southeast (Rio de Janeiro and Queimados) and 1 located in the Northeast region of Brazil (Fortaleza). All plasma samples were tested for anti-HEV IgG antibodies detection by ELISA and tested for HEV-RNA detection by RT-qPCR for ORF3 region amplification.

Results: General anti-HEV prevalence was $24.48 \%$ (70/286 positive for anti-HEV IgG antibodies) with different local prevalences of $37.80 \%$ in Rio de Janeiro, $0 \%$ in Queimados and $27.27 \%$ in Fortaleza. Lower family income and longer period of time under hemodialysis were statistically related to anti-HEV IgG positivity. Concerning biochemical analysis, glicose levels were also related to anti-HEV IgG+ samples $(\mathrm{p}=0)$, iron levels were lower in anti-HEV IgG+ samples, suggesting a role of HEV proteins in iron metabolism modulation. No samples were for HEV RNA detection.

Conclusion: This study corroborates previous studies that show a higher prevalence in HD patients in comparison to health patients and reinforce the importance of monitoring these patients.

Keywords: HEV; chronic kidney disease; prevalence 